\title{
Improving the quality of spatial separation of signals in radio communication systems
}

\author{
Aleksandr I. Okorochkov ${ }^{1, *}$, Bogdan B. Malyutin ${ }^{1}$, Mohamed Amine Laouar ${ }^{2}$, and \\ Nadezhda A. Dmitrienko ${ }^{1}$ \\ ${ }^{1}$ Don State Technical University, Institute of Service and Business (Branch) Don state technical \\ university (DSTU) in Shakhty, 346500 Shakhty Shevchenko 147, Russian Federation \\ ${ }^{2}$ Abbas Laghrour University, 40000 Khenchela, Hamma 1, Algeria
}

\begin{abstract}
The article presents the research results of dependence of the signal-to-interference ratio in the focus area of the reflecting antenna array on its length, as well as the influence of the center displacement of the antenna array of different sizes on the signal-to-interference ratio. The research results show that short-focus reflecting antenna arrays have better characteristics than long-focus ones. By displacing the center of the antenna array, the signal-to-interference ratio can be increased by almost $20 \mathrm{~dB}$.
\end{abstract}

\section{Introduction}

To increase the capacity of radio channels, various methods are used, including the organization of parallel channels in one frequency band. This approach allows more efficient use of scarce frequency resource. Let different signals in one frequency channel be transmitted at the same time, in the same direction and with the same field polarization. Then the only parameter by which they can be distinguished at the reception is the spatial structure of the complex amplitudes of the fields of these signals.

In [1] the structure of the communication system with the separation of two signals at the reception of the curvature of the wave fronts of their fields is proposed. A linear sparse antenna array (SAA) consisting of three parabolic antennas was used as a transmitting antenna. Each of the transmitted signals was emitted by a different number of antennas. This provided a different curvature of the wave fronts of their fields. The signals from the outputs of each receiving antenna were processed separately using an adaptive spatial processing algorithm $[2,3]$. As a result of such processing, one of the amplified signals was extracted at the input of the first receiver, and the second signal was significantly suppressed. At the input of the second receiver, the ratio of signal levels was reversed. The evaluation of the properties of such a communication system [4] showed that, depending on the degree of difference of wave fronts, it is possible to achieve the signal/(interference + noise) ratio at the level of $31 \ldots 39 \mathrm{~dB}$. The degree of difference in curvature of wave fronts can be adjusted only by the number of antennas emitting each signal. In addition, the curvature of the wave fronts of signals is most pronounced only in the intermediate zone of

${ }^{*}$ Corresponding author: okorochkov_a@mail.ru 
the radiation field, which limits the communication range in such systems. It should be noted that the analysis of the properties of the considered communication system was carried out for narrowband signals in the space-time sense $[5,6]$.

In papers [7], [8], [9], another method of spatial separation of signals at the reception was proposed, free from limitations of the communication system considered earlier. The method consists in forming in the location of receiving antennas such an optimal structure of the fields of two transmitted signals (Fig. 1), in which the maximum amplitude of the field of one signal (useful) and zero of the amplitude of the field of another signal (interference) are combined at one point of space.

In Fig. 2 the diagrams of the phase distribution of the transmitted signals along the line parallel to the line of transmitting antennas (perpendicular to the communication direction) are given. It can be seen from them that at the zero point of the amplitude of any signal its phase changes abruptly by $180^{\circ}$, and in limited areas to the right and left of this point of the phase, the amplitudes of the signal is almost opposite. This allows combining the center of the aperture of the receiving antenna with the zero point of the amplitude of the noise to get its output amplified useful signal. The interference signal will be drastically weakened due to the mutual compensation of its anti-phase components during their coherent addition at the aperture of the receiving antenna. The estimates show that in this case the signal-tointerference ratio (SIR) level reaches $59 \ldots 62 \mathrm{~dB}$.

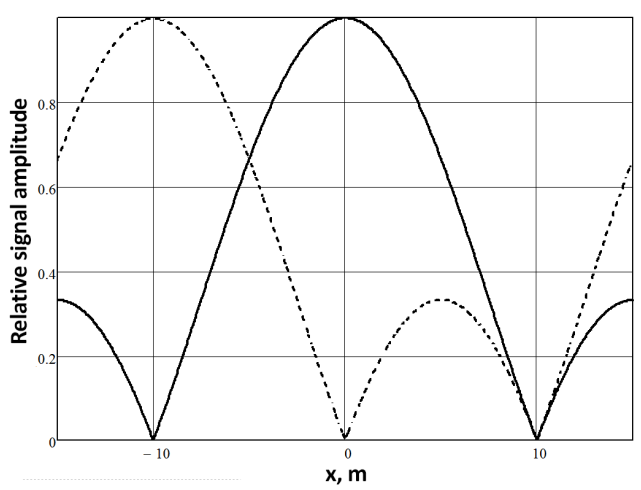

Fig. 1. Amplitude distribution of the fields of two signals in the receiving plane.

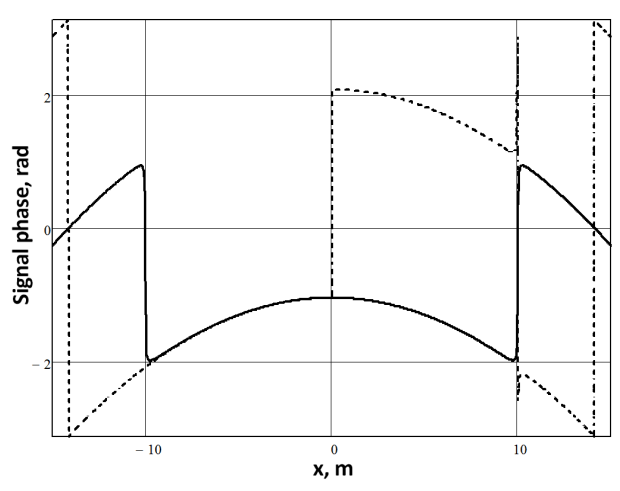

Fig. 2. Phase distribution of the fields of two signals in the receiving plane.

In the discussed communication system, linear SAA of three aperture antennas was used as a transmitting antenna system. In addition, each of the two signals was transmitted by all three antennas.

The components of each signal at the inputs of all antennas were coherent with each other, since they were formed from a single source. The amplitude and phase for each signal at the input of each antenna was set individually. The selection of these parameters allowed obtaining the optimal structure of the field in the area of receiving antennas. The receiving antenna had a complex structure and consisted of a linear reflecting antenna array (RAA) and the receiving aperture antenna itself as a horn. The linear RAA performed a complex function - it reflected both signals and focused them into the area of the aperture of the receiving antenna. Focusing made it possible to raise the level of the useful signal even higher, and the interference was still suppressed due to the establishing two anti-phase components on the receiving aperture of its amplitude.

Thus, it was possible to increase SIR to $\sim 67 \mathrm{~dB}$. The analysis of the properties of the last communication system, as well as for the previous one, was carried out for narrowband signals in the space-time sense. 


\section{Defining research objectives}

The analysis of the graphs of distribution of the amplitude and phase of the signal fields along the axis of the linear RAA in Fig. 1 and Fig. 2 shows that when a linear RAA is located symmetrically to the zero point of the signal-interference amplitude, there exists two reasons that prevent to establish the maximum possible SIR in the communication system under consideration:

- asymmetrical behavior of the amplitude of the signal-interference graph on both sides of the zero point amplitude (with an odd number of transmitting antennas), which does not allow to achieve maximum compensation of the anti-phase amplitude components of this signal at the receiving antenna aperture;

- weak anti-phase of components of the signal-interference, falling on the sections of RAA to the right and left of the point of zero amplitude of this signal.

This article discusses the way of eliminating restrictions on the level of SIR paying attention to the first of the pointed reasons. The aim of the research is to determine the dependence of SIR on the size and position of the linear RAA and obtain the optimal values of these parameters at which the best SIR is achieved. The research was carried out by mathematical modeling.

\section{Description of the mathematical model of the communication channel}

The aim was achieved by modeling wave processes in a communication system with certain values of its parameters and the following simplifying restrictions:

- as a model of the radiation field of one transmitting antenna, the radiation field of a round flat aperture with a radius of $a=0.5 \mathrm{~m}$ and a uniform amplitude and phase distribution over the aperture was chosen;

- the resulting signal field in the receiving area was obtained by scalar summing the fields of all the antennas emitting the signal;

- the propagation medium of radio waves was considered homogeneous and isotropic, and the propagation conditions in the space of the fields of all transmitting antennas were considered the same;

- the multipath nature of radio wave propagation was not considered;

- the transmitting antenna system was a linear SAA of three round flat apertures with distances between two adjacent apertures $h=10 \mathrm{~m}$;

- the carrier frequency of the radiation field was $15 \mathrm{GHz}$ (wavelength is $\lambda=2 \mathrm{~cm}$ ), and the communication range is $d=15 \mathrm{~km}$;

- a model of a narrowband signal in the space-time sense was used, which made it possible to separate the temporal and spatial parts of the signal in the model and simplify the analysis of its spatial properties

- random errors of amplitude-phase distribution in the aperture of the transmitting SAA were not taken into account;

- sources of radio emission were considered, because conditions are met to: $a, h<<d$;

- RAA consisted of a set of $1 \times 1 \mathrm{~cm}$ diffractive elements located along a straight line with a step $\lambda$ [8]; a separate diffraction element had the structure of a strip emitter, geometrical dimensions of which were selected to provide a specified phase shift in the field during reflection; on the whole, the phasing of all diffractive elements ensured the focusing of the reflected field at a given point of the focus; the amplitude pattern of the 
diffraction element was obtained using electro dynamical modeling and approximated by the formula $F=\cos ^{3} \theta$, where $\theta$ is the angle between the direction of the radiation maximum and the observation point (in the horizontal plane);

- the spatial structure of the radiation field in the reception area was analyzed only along a line parallel to the axis of the linear radiating SAA;

- the power of the useful signal and interference at the input of the receiving antenna was estimated by integrating the square of the module of the complex amplitude of the total field of each signal over the aperture of the receiving antenna located in the focus area of the reflected signal.

\section{Order and results of simulation}

Studies were conducted in two stages. At the first stage, dependences of the levels of the useful signal, interference and SIR on the length of the RAA for two values of the focal distance $\mathrm{F} 1=2 \mathrm{~m}$ and F2 $=5 \mathrm{~m}$ were determined. The length of the RAA is symmetrically increased at both ends by adding diffraction elements. The center of the RAA was not shifted and was at the zero point of the amplitude of the signal-interference field. The focal point was against the center of the RAA. The simulation results are presented in Fig. 3, 4 and 5 .

Analysis of graphs in Fig. 3, 4 and 5 allows us to come to the following conclusions in favor of using RAA with a shorter focal length $\mathrm{F} 1=2 \mathrm{~m}$ :

- the maximum level of the useful signal is higher and is achieved with a smaller length of the RAA;

- with a number of elements of RAA $n<200$, the level of interference in the RAA with different focal distances is almost the same. However, for $n>200$, the level of interference in a longer-focus system increases noticeably faster.

The first advantage of a shorter focus RAA is due to two reasons:

- while maintaining the length of the RAA, an increase in the focal distance is accompanied by an increase in the attenuation of the useful signal along the path from the RAA to the focus point;

- if you increase the length of the antenna with an increase in focal distance, then a significant compensation of losses will not work. This is explained by a decrease in the contribution of the fields of RAA elements located closer to the edges of the array to the total field in focus. The reason for this decrease is the lower level of the useful signal field in the area of the extreme elements, as well as the shape of their amplitude radiation pattern.

The second advantage of the short-focus RAA is due to the fact that with increasing the length of RAA, the cut-off of the contribution of the extreme elements of the short-focus array occurs earlier than in the long-focus array. In this case, a greater number of extreme elements of the long-focus array fall within the signal-interference field with the opposite phase and different amplitude. The growth of the differential signal interference on the aperture of the receiving antenna and is the cause of a more rapid increase in the level of interference in long-focus antenna systems.

As a result, summing up the analysis that has been carried out, it can be concluded that short-focus RAAs have advantages over long-focus ones in all considered indicators.

At the second stage, the possibility of increasing SIR due to the shift of the center of the RAA from the zero point of the amplitude of the noise towards its smaller maximum was investigated (in Fig. 1, this shift is to the right). Such shift can contribute to better compensation of the anti-phase components of the amplitude of the interference on the 
receiving antenna aperture and thereby further increase of SIR. This study was conducted as follows.

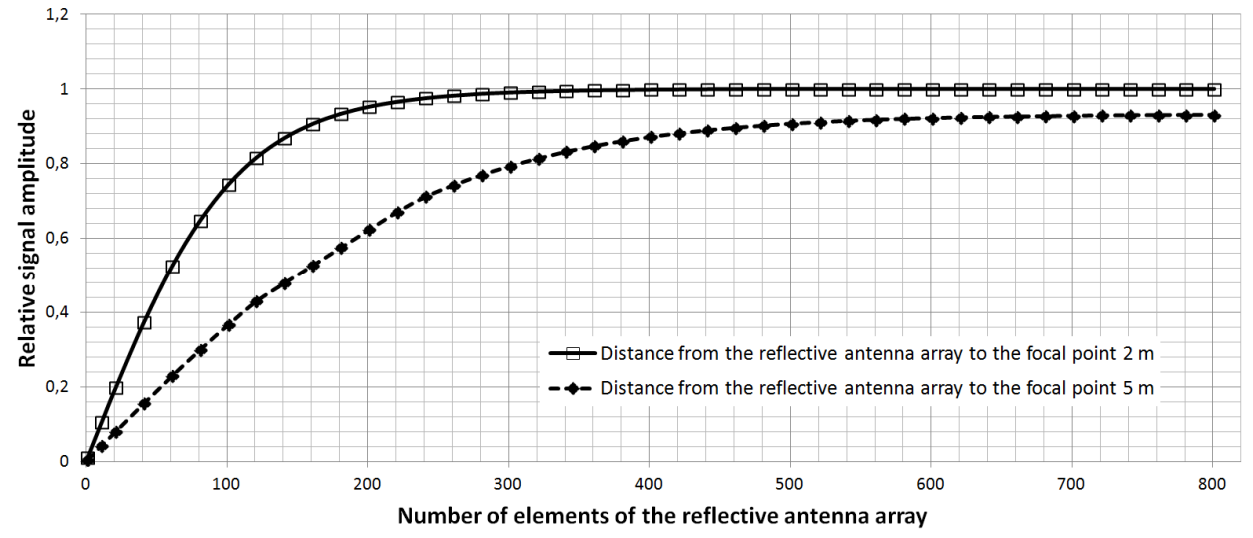

Fig. 3. Dependences of the normalized amplitude of the signal on the number of elements of the RAA for two values of the focal distance $\mathrm{F} 1=2 \mathrm{~m}$ and $\mathrm{F} 2=5 \mathrm{~m}$.

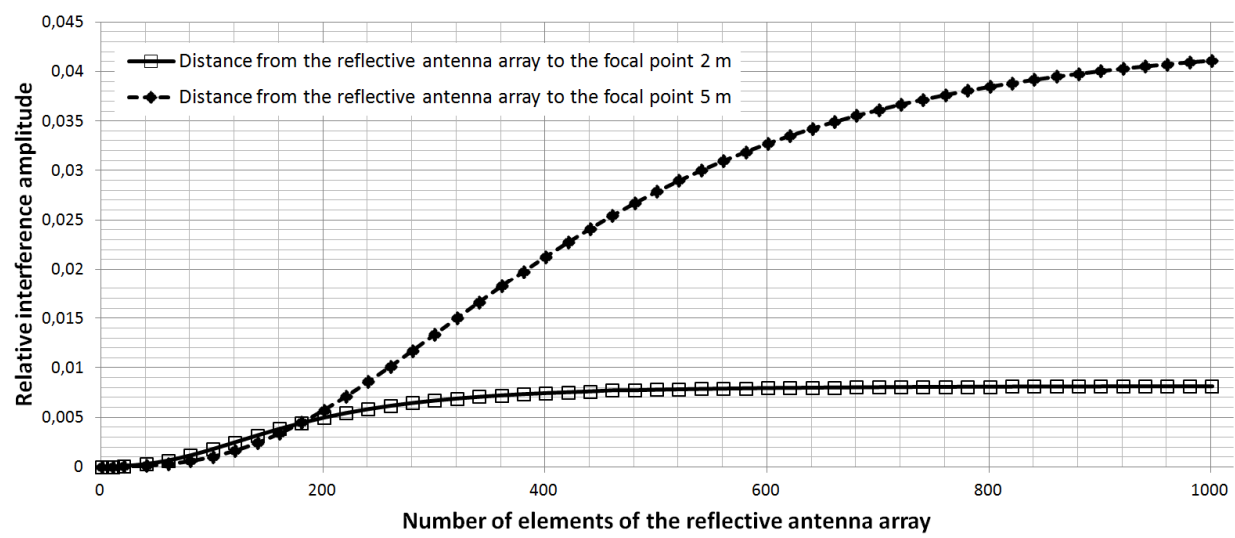

Fig. 4. The dependences of the normalized amplitude of interference on the number of elements of the RAA for two values of the focal distance F1 $=2 \mathrm{~m}$ and F2 $=5 \mathrm{~m}$.

Firstly, one diffraction element of the RAA was installed at the zero point of the amplitude of the signal-interference. In this case, the relative level of the useful signal $S$, SIR, the X coordinate of the center of the RAA and its length (number of elements) were estimated and recorded. Next, a symmetric expansion of the RAA was performed (by adding the number of diffraction elements) without shifting of its center until SIR is decreased by about $10 \mathrm{~dB}$. Then, the entire RAA is shifted to the right from the zero point of the amplitude of the signal-interference to a position at which SIR can reach its maximum. After that, new values of the parameters pointed above were recorded and the procedure was repeated. It should be noted that the focal point is shifted along with the center of the RAA. The value of the focal distance was taken $\mathrm{F} 1=2 \mathrm{~m}$ for the reasons stated above. The results are shown in Fig. 6

These results confirm the initial assumption that by using the displacement of the center of the RAA, it is possible to achieve the best SIR. And indeed, as follows from the graph in Fig. 6, the initial level of SIR, is equal to $67 \mathrm{~dB}$, in certain positions of the center of the RAA it is exceeded by $15 \ldots 20 \mathrm{~dB}$. 


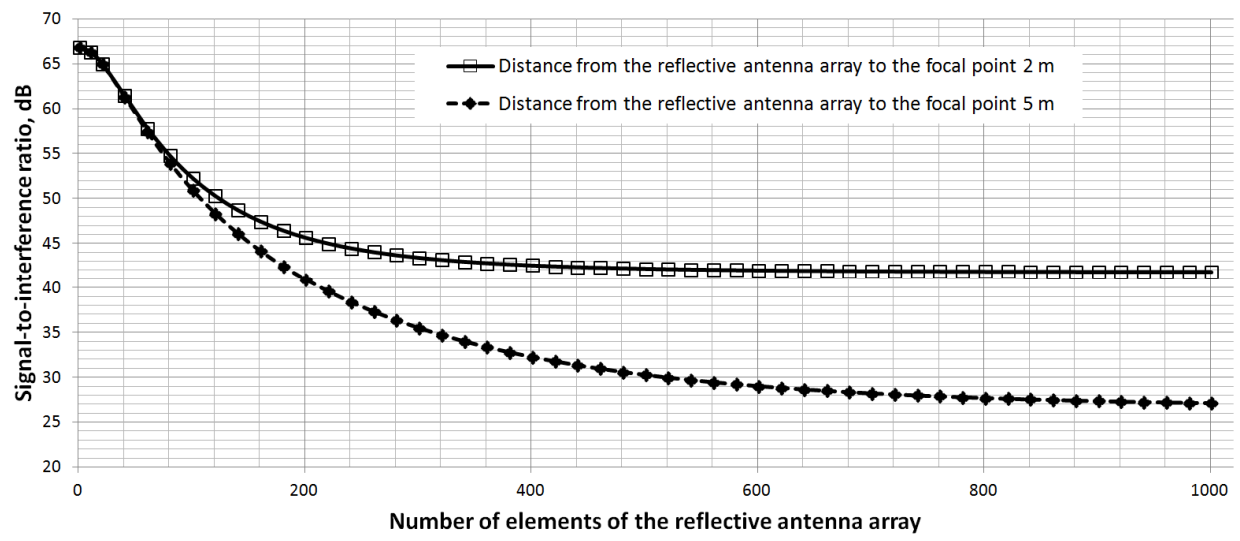

Fig. 5. Dependencies of signal-to-noise ratio on the number of RAA elements for two values of focal distance $\mathrm{F} 1=2 \mathrm{~m}$ and $\mathrm{F} 2=5 \mathrm{~m}$.

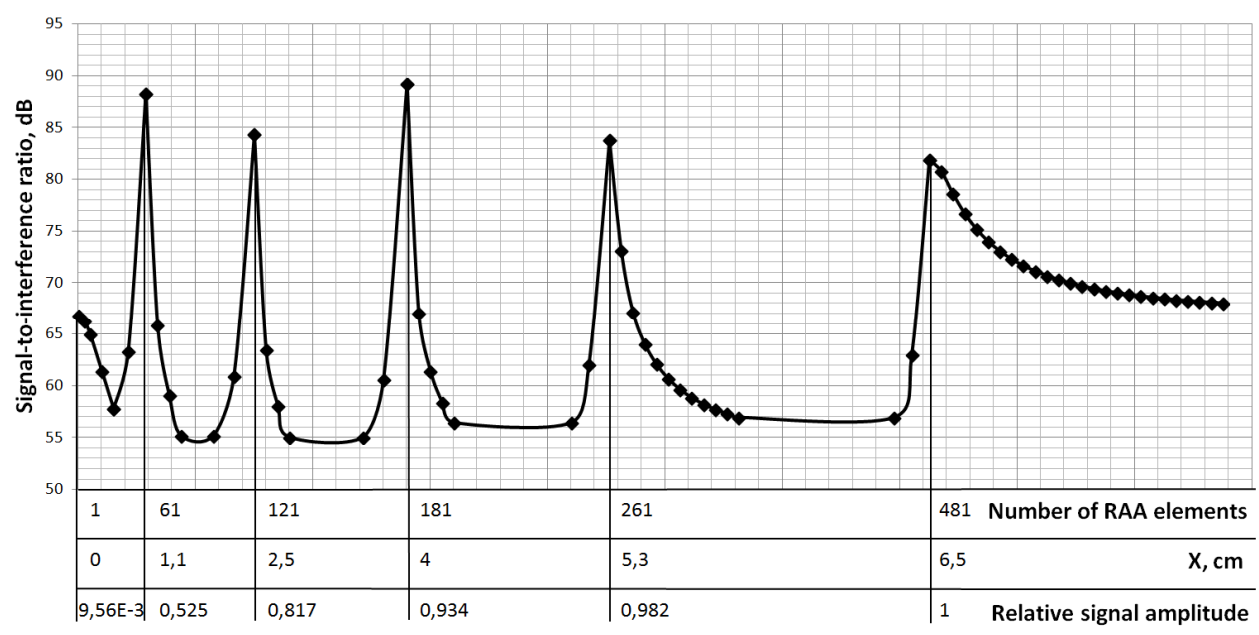

Fig. 6. The influence of the length of the RAA and its center shift on the signal-to-noise ratio and the level of the useful signal at the focal distance F1.

It should be noted that there are very slight shifts of the center of the RAA (several centimeters), corresponding to the maxima of SIR, with a total length of the RAA of several meters. The narrowness of the zones in which the maximum SIR is reached will require, in practice, high accuracy in positioning the RAA relative to the spatial structure of the radiation field. It is also interesting to note the fact that the maximum SIR is achieved at different levels of the useful signal $S$. The reserve in terms of the useful signal is necessary, since in the receiver, noise will be added to the noise and the overall signal / (interference + noise) ratio will be decreased.

\section{Conclusion}

Summing up the research we can come to the following conclusions:

- spatial separation of signals, contributing to an increase in the bandwidth of the communication channel and more efficient use of its frequency resource, requires a comprehensive and in-depth study of the conditions for its provision and effective use; 
- a careful analysis of the conditions of forming the spatial structure of the radiation field made it possible to find additional reserves for increasing SIR - this is the asymmetry of the graph of the amplitude of the interference field in the vicinity of its amplitude zero point and weak anti-phase interference signal in the same neighborhood;

- when constructing RAA with focusing of a reflected field, arrays with a shorter focus should be used, since they have the best set of parameters;

- the application of displacement of the center of the linear RAA offset in the horizontal direction from zero point of the signal-interference amplitude towards the small maximum of the amplitude of this signal (Fig. 1) allows further increasing SIR by $15 \ldots 20 \mathrm{~dB}$.

\section{References}

1. E.A Yakornov, M.A. Kolomytsev, G.L. Avdeenko, O.Y. Lavrinenko, Visnik Natsional'nogo tekhnichnogo universitetu Ukraïni "KPI" Seriya - Radiotekhnika. Radioaparatobuduvannya [Bulletin of the National Technical University of Ukraine "KPI" Series - Radio Engineering. Radio equipment construction], 48, 97 (2012) (in Russian)

2. R.A. Monzingo, T.W. Miller, Adaptive antenna arrays. Introduction to the theory (Radio i Svyaz', Moscow, 1986) (in Russian)

3. J.R. Guerci. Space-Time Adaptive Processing for Radar (Artech House, Norwood, MA, USA, 2003)

4. G.L. Avdeenko, E.A. Yakornov, Visnik Natsional'nogo tekhnichnogo universitetu Ukraïni "KPI" Seriya - Radiotekhnika. Radioaparatobuduvannya [Bulletin of the National Technical University of Ukraine "KPI" Series - Radio Engineering. Radio equipment construction], 52, 92 (2013) (in Russian)

5. I.Y. Kremer, A.I. Kremer, V.M. Petrov, V.A. Pon'kin, N.A. Potapov. Prostranstvennovremennaya obrabotka signalov [Spatial-time signal processing]. (Radio i Svyaz' Publ., Moscow, 1984) (in Russian)

6. R. Klemm (ed.) Applications of Space-Time Adaptive Processing (IEE, London, 2004)

7. A.I. Okorochkov, G.L. Khripkov, Proc. Conf. Nauchnaya vesna - 2017: Tekhnicheskie nayki [Scientific spring 2017: Technical science], 314 (2017) (in Russian)

8. A.I. Okorochkov, B.B. Malyutin, Proc. Conf. Nauchnaya vesna - 2017: Tekhnicheskie nayki [Scientific spring 2017: Technical science], 308 (2017) (in Russian)

9. A.I. Okorochkov, B.B. Malyutin, MATEC Web Conf, 226, 05010 (2018) 